\title{
The Stability of Difference Approximations to a Self-Adjoint Parabolic Equation, Under Derivative Boundary Conditions
}

\author{
By C. M. Campbell and P. Keast
}

1. Introduction. A self-adjoint parabolic equation in one space variable is considered, under boundary conditions which involve the function and its space derivative. A type of numerical instability can arise, which is traceable to the boundary conditions, and which is caused by the existence of unbounded solutions of the original differential equation.

2. The Differential Equation. The equation to be examined is

$$
\frac{\partial u}{\partial t}=\frac{\partial}{\partial x}\left(g(x) \frac{\partial u}{\partial x}\right)
$$

in the region

$$
R \equiv[a \leqq x \leqq b] \times[t \geqq 0]
$$

subject to the initial condition

$$
u(x, 0)=y(x), \quad a \leqq x \leqq b,
$$

and the boundary conditions

$$
\begin{array}{ll}
\partial u / \partial x-p u=\phi_{0}(t), & x=a, \quad t \geqq 0, \\
\partial u / \partial x+q u=\phi_{1}(t), & x=b,
\end{array}
$$

where $p, q$ are constants, $\phi_{0}(t)$ and $\phi_{1}(t)$ are bounded as $t \rightarrow \infty$, and there are no discontinuities in the initial or boundary conditions, or at the corners of $R$. In addition, we assume $g(x)>0$, for $a<x<b$.

Several authors (e.g. [1]-[5]) have considered the equation (1) subject to (1a) and (1b) for the particular case when $p \geqq 0$ and $q \geqq 0$. These conditions are not imposed in this paper. In [6] Keast and Mitchell have considered the problem of the present paper with $g(x) \equiv 1$.

3. The Difference Approximations. The region $R$ is covered by a rectangular mesh, the nodal points of which are given by

$$
x_{i}=a+i h ; \quad i=0,1, \cdots, N ; \quad N h=b-a
$$

and

$$
t_{n}=n k, \quad n \geqq 0 .
$$

The constants $h$ and $k$ are the space and time increments respectively, and the ratio $k / h^{2}$ is denoted by $r$. 
We consider an approximation to Eq. (1) which can be written in the form (2) $w_{i}{ }^{n+1}-w_{i}{ }^{n}=\frac{r}{2} \Delta\left(B_{i} \nabla w_{i}{ }^{n}\right)+\frac{r}{2} \Delta\left(B_{i} \nabla w_{i}{ }^{n+1}\right), \quad i=0,1, \cdots, N ; n \geqq 0$, where $w_{i}{ }^{m}(m=n, n+1)$ is the numerical approximation to $u\left(x_{i}, t_{m}\right)$; the difference operators $\Delta$ and $\nabla$ are defined by

$$
\Delta f_{i}=f_{i+1}-f_{i} \quad \text { and } \quad \nabla f_{i}=f_{i}-f_{i-1},
$$

and the coefficients $B_{i}(i=0,1, \cdots, N+1)$ are defined, using a method developed by Tikhonov and Samarskii [7], by

$$
B_{i}=\left[\int_{x_{i-1}}^{x_{i}} \frac{d x}{g(x)}\right]^{-1}, \quad i=0,1, \cdots,(N+1) .
$$

In Eq. (2), when $n=0$, the values of $w_{i}{ }^{0} \quad(i=0,1, \cdots, N)$ are obtained from the initial conditions (1a). When $i=0$ or $N$, the values of $w$ outside $R$, viz. $w_{-1}^{m}$ and $w_{N+1}^{m},(m=n, n+1)$, which occur in (2) are eliminated using the boundary conditions $(1 \mathrm{~b})$, in which the derivatives are approximated by the equation

$$
\partial u_{j}^{m} / \partial x=\left(w_{j+1}^{m}-w_{j-1}^{m}\right) / 2 h, \quad j=0, N \text { and } m=n, n+1 .
$$

If we denote the vector of values of $w$ at each time level $t_{m}$ by $\mathbf{w}^{m}$, i.e.

$$
\mathbf{w}^{m}=\left\{w_{0}{ }^{m}, w_{1}{ }^{m}, \cdots, w_{N}{ }^{m}\right\}^{T}
$$

and use the boundary conditions (1b) in (2) when $i=0$ and $i=N$, we may write the totality of difference equations in the form

$$
(I+r U / 2) \mathbf{w}^{n+1}=(I-r U / 2) \mathbf{w}^{n}+l^{n}
$$

where $1^{n}$ is a vector involving the boundary conditions (with $l_{1}{ }^{n}=l_{2}{ }^{n}=\cdots$ $=l_{N-1}^{n}=0$, for all $\left.n\right)$ and $I$ and $U$ are matrices of order $(N+1)$. The matrix $I$ is the identity matrix, and $U$ is given by

(7)

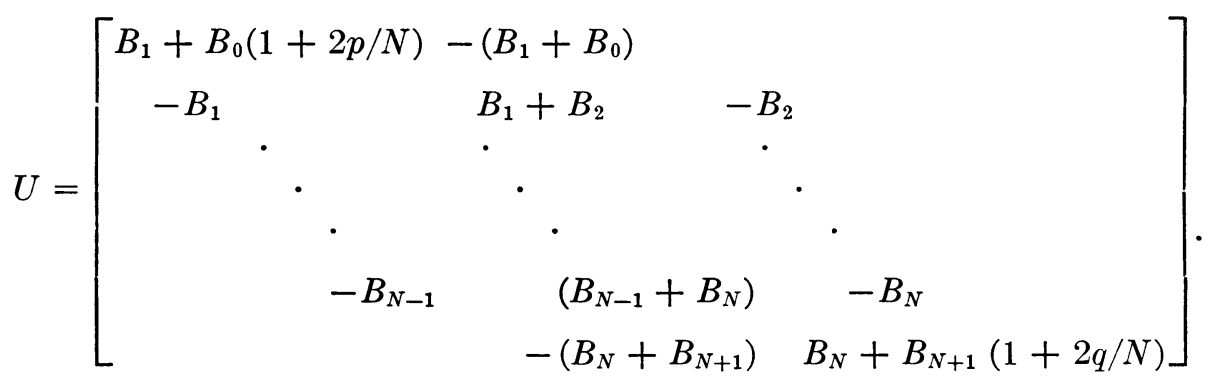

Let the numerical error of the difference method (6) be denoted by

$$
\boldsymbol{\varepsilon}^{n}=\mathbf{w}^{n}-\hat{\mathbf{w}}^{n}, \quad \forall n \geqq 0,
$$

where $\mathbf{w}^{n}$ is the exact solution of Eq. (6) and $\hat{\mathbf{w}}^{n}$ is the numerical solution. Then we obtain the error equation

$$
(I+r U / 2) \varepsilon^{n+1}=(I-r U / 2) \varepsilon^{n} .
$$

We shall assume $(I+r U / 2)$ to be nonsingular, so that 
(8)

$$
\boldsymbol{\varepsilon}^{n+1}=(I+r U / 2)^{-1}(I-r U / 2) \varepsilon^{n} .
$$

The difference method (6) will be stable, therefore, if and only if the norms $\left\|\varepsilon^{n}\right\|$ of the vectors given by (8) are bounded. Since

$$
\varepsilon^{n+1}=G^{n+1} \varepsilon^{0}
$$

where

$$
G=(I+r U / 2)^{-1}(I-r U / 2)
$$

then the norms $\left\|\varepsilon^{n}\right\|$ are bounded if and only if there is a constant $K$ such that

$$
\left\|G^{n+1}\right\| \leqq K, \quad \forall n \geqq 0 .
$$
where

The matrix $U$ is not symmetric, but it is similar to a symmetric matrix $\tilde{U}$,

$$
\tilde{U}=D^{-1} U D
$$

with $D$ given by

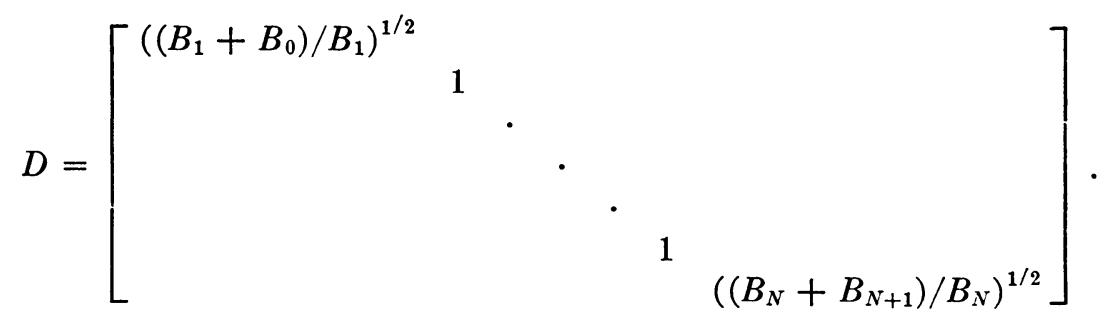

Hence the eigenvalues of $U$ are all real.

Thus $G$ is similar to a symmetric matrix $\widetilde{G}$, where

$$
\widetilde{G}=D^{-1} G D
$$

and if $\rho(G)$ is the spectral radius of $G$ (the maximum modulus eigenvalue) then

$$
\left\|\widetilde{G}^{n+1}\right\|=\rho^{n+1}(G) \text {. }
$$

Clearly $\left\|G^{n+1}\right\|$ is bounded if and only if $\left\|\widetilde{G}^{n+1}\right\|$ is bounded. The stability condition is therefore: there exists $K>0$, for all $n>0, \rho^{n+1}(G) \leqq K$.

This condition clearly implies

$$
\rho(G) \leqq 1
$$

It should be noted that condition (10) is the condition for asymptotic stability $(n \rightarrow \infty)$ of Eq. (6). If the errors are to be bounded in a closed region $0 \leqq t \leqq T$, for some finite $T$, then (10) may be weakened to the condition

$$
\rho(G) \leqq 1+O(k) \text {. }
$$

This point has been discussed by Gary [8]. In this paper we take (10) as our condition for stability.

If we denote the $(N+1)$ real eigenvalues of the matrix $U$ by $\lambda_{0}, \lambda_{1}, \cdots, \lambda_{N}$, then condition (10) is satisfied if and only if

$$
\left|\left(1-r \lambda_{i} / 2\right) /\left(1+r \lambda_{i} / 2\right)\right| \leqq 1, \quad i=0,1, \cdots, N .
$$


We shall assume that $1+r \lambda_{i} / 2>0$ (an assumption which is justified later) and hence the necessary and sufficient condition for stability becomes

$$
\lambda_{i} \geqq 0, \quad i=0,1, \cdots, N .
$$

4. The Eigenvalues of $U$. If we apply the theorem of Gerschgorin to the matrix $U$ in (7), we find that the spectrum of $U$ lies within the union of the intervals

$$
\begin{gathered}
0 \leqq \lambda \leqq 2\left(B_{i}+B_{i+1}\right), \quad i=1,2, \cdots,(N-1) \\
\left(2 B_{0} / N\right) p \leqq \lambda \leqq 2\left[B_{0}(1+p / N)+B_{1}\right] \\
\left(2 B_{N} / N\right) q \leqq \lambda \leqq 2\left[B_{N+1}(1+q / N)+B_{N}\right] .
\end{gathered}
$$

If $p \geqq 0$ and $q \geqq 0$ then $U$ is positive definite (or positive semidefinite if $p=q=0$ ), and Eq. (6) is then stable. This is the case which has been considered by the authors [1]-[5]. If any eigenvalues of $U$ are negative, then they are $O(1 / N)$, and so for large $N$ we may assume that $1+r \lambda_{i}>0, i=0,1, \cdots, N$. This justifies the assumption made in Section 3.

We now consider for what values of $p$ and $q$ the matrix $U$ is singular, i.e. $\operatorname{det}(U)=0$. Expanding this determinantal equation we obtain

$$
\begin{aligned}
& \left(4 p q / N^{2}\right) B_{0} B_{N+1} T_{N-1}^{(1)}+(2 p / N) B_{0}\left(B_{N}+B_{N+1}\right)\left(T_{N-1}^{(1)}-B_{N} T_{N-2}^{(1)}\right) \\
& \quad+2(q / N) B_{N+1}\left(B_{0}+B_{1}\right)\left(T_{N-1}^{(1)}-B_{1} T_{N-2}^{(2)}\right) \\
& \quad+\left(B_{0}+B_{1}\right)\left(B_{N}+B_{N+1}\right)\left(T_{N-1}^{(1)}-B_{N} T_{N-2}^{(1)}-B_{1} T_{N-2}^{(2)}+B_{1} B_{N} T_{N-3}^{(2)}\right)=0
\end{aligned}
$$

where

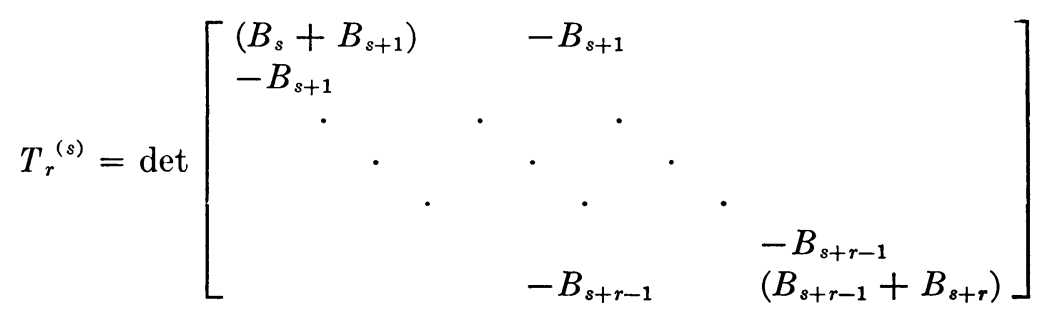

is the determinant of an $(r \times r)$ symmetric matrix. The constant term (i.e. the term independent of $p$ and $q$ ) in (11) is the expansion of det $U$ when $p=q=0$. But if $p=q=0, U$ is singular (the sum along each row is zero), and so this constant is zero.

It is easily shown that

$$
T_{r}^{(s)}=\sum_{j=0}^{r}\left\{\prod_{i=s ; i \neq j+s}^{r+s} B_{i}\right\}
$$

and hence that

$$
T_{N-1}^{(1)}-B_{N} T_{N-2}^{(1)}=\prod_{i=1}^{N-1} B_{i}
$$

and

$$
T_{N-1}^{(1)}-B_{1} T_{N-2}^{(2)}=\prod_{i=2}^{N} B_{i} .
$$


Equation (11) then becomes

$$
\begin{aligned}
\frac{4 p q}{N^{2}} B_{0} B_{N+1} \sum_{j=0}^{N-1} \prod_{i=1 ; i \neq j+1}^{N} B_{i}+\frac{2 p}{N} \frac{\left(B_{N}+B_{N+1}\right)}{B_{N} B_{N+1}} \prod_{i=0}^{N+1} B_{i} \\
+\frac{2 q}{N} \frac{B_{0}+B_{1}}{B_{0} B_{1}} \prod_{i=0}^{N+1} B_{i}=0 .
\end{aligned}
$$

This may be rearranged to give

$$
\frac{4 p q}{N^{2}} \sum_{i=1}^{N} \frac{1}{B_{i}}+\frac{2 p}{N} \frac{B_{N}+B_{N+1}}{B_{N} B_{N+1}}+\frac{2 q}{N} \frac{B_{0}+B_{1}}{B_{0} B_{1}}=0 .
$$

For the method of Tikhonov and Samarskii this equation takes the form

$$
\frac{4 p q}{N^{2}} \int_{a}^{b} \frac{d x}{g(x)}+\frac{2 p}{N} \int_{x_{N-1}}^{x_{N+1}} \frac{d x}{g(x)}+\frac{2 q}{N} \int_{x_{-1}}^{x_{1}} \frac{d x}{g(x)}=0 .
$$

It should be noted that this is an approximation, to $O\left(1 / N^{2}\right)$, of the equation

$$
p q \int_{a}^{b} \frac{d x}{g(x)}+\frac{p}{g(b)}+\frac{q}{g(a)}=0 .
$$

The matrix $U$ is singular when $p$ and $q$ lie on a hyperbola, which we denote by $\Sigma=0$, where

$$
\Sigma \equiv p q+L p+M q=0 .
$$

The coefficients $L, M$ are both positive. We partition the $(p, q)$ plane into sections $A, B, D$ where
(i) $A$ is $p q+L p+M q \geqq 0, p+q \geqq 0$,
(ii) $B$ is $p q+L p+M q<0$,
(iii) $D$ is $p q+L p+M q \geqq 0, p+q<0$, (see Fig. 1).

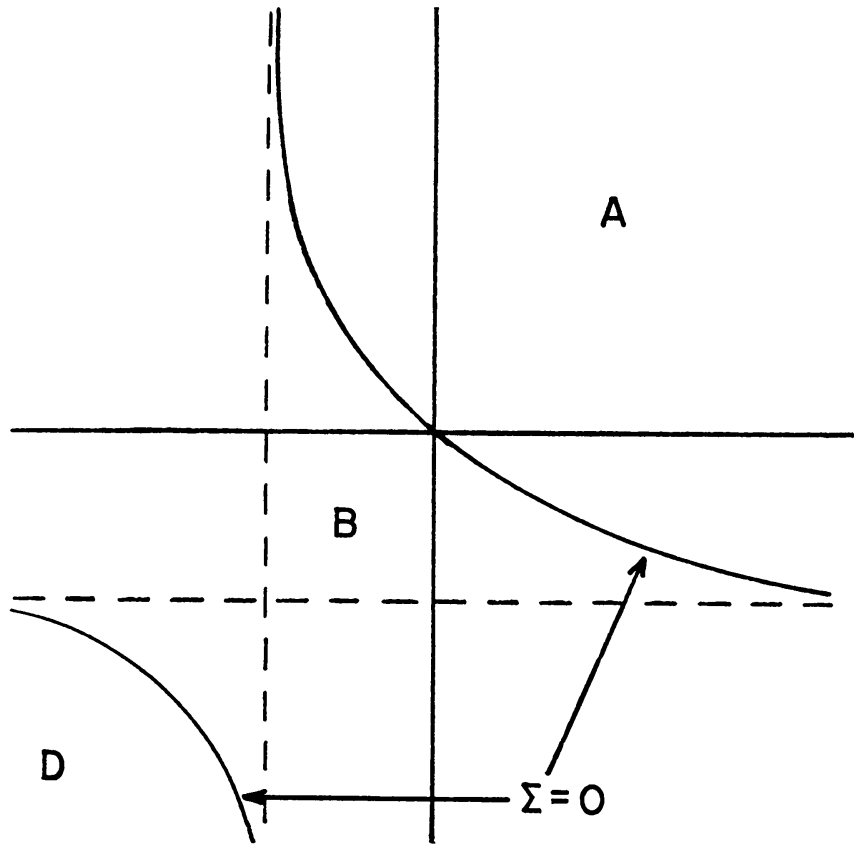


Let $z(\lambda)$ be the characteristic polynomial of the matrix $U$, i.e.

$$
z(\lambda) \equiv \operatorname{det}(U-\lambda I) .
$$

Since $U$ is similar to a symmetric matrix, the polynomial $z(\lambda)$ has $(N+1)$ real zeros; in addition $z(\lambda)$ is clearly positive for large negative $\lambda$. But in the region $B, z(0)$ is negative, and so there must be a zero of $z(\lambda)$ in $\lambda<0$. In fact $z(\lambda)$ must have an odd number of negative roots. At the point $p=-N / 2, q=-N / 2$ in $D$ the matrix $U$ has at least one negative root, since

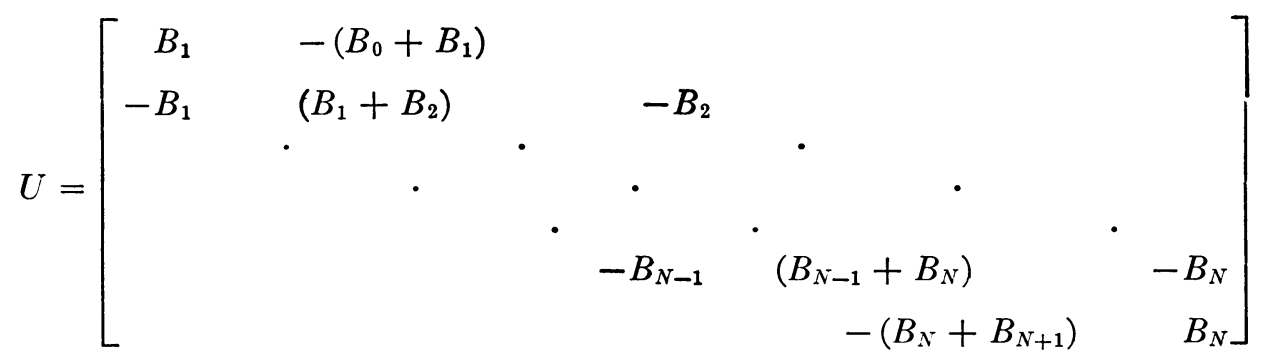

which is not positive definite, since

$$
U\left[\begin{array}{c}
1 \\
1 \\
\vdots \\
1
\end{array}\right]=\left[\begin{array}{c}
-B_{0} \\
0 \\
0 \\
\vdots \\
0 \\
-B_{N+1}
\end{array}\right]<0
$$

Thus, since $z(-\infty)>0$ and $z(0)>0$ in $D$, there is an even number of negative roots at the point $(-N,-N)$.

We now exhibit the dependence of $z(\lambda)$ on $(p, q)$ by writing the characteristic polynomial as $z(p, q, \lambda)$. The function $z=0$ is a surface in $(p, q, \lambda)$ space, cutting each vertical line $p=$ constant, $q=$ constant, in $(N+1)$ points. Let $\Gamma(p, q)=0$ be any curve in the $(p, q)$ plane. Let $\Gamma_{\lambda_{0}}=0$ be the curve in $(p, q, \lambda)$ space, on the surface $z=0$, which consists of the points $\left(p, q, \lambda_{0}(p, q)\right)$, where $\lambda_{0}$ is the smallest root of $z=0$, for each point $(p, q)$ on $\Gamma(p, q)=0$. Thus $\Gamma=0$ is the projection of $\Gamma_{\lambda_{0}}=0$ on the $(p, q)$ plane. Clearly, as $(p, q)$ moves, the curve $\Gamma_{\lambda_{0}}=0$ is continuous. Suppose that $(p, q)$ moves from a point in the region $A$ where $p>0$ and $q>0$, along $\Gamma=0$. Then initially $\lambda_{0}>0$, and as $(p, q)$ moves, $\lambda_{0}$ cannot change sign unless $\Gamma_{\lambda_{0}}=0$ crosses the $(p, q)$ plane; i.e. unless $\lambda_{0}$ passes through a zero. This cannot happen unless $\Gamma_{\lambda_{0}}=0$ and $\Gamma=0$ pass through a point on $\Sigma=0$ in the $(p, q)$ plane. Thus at every point in $A, \lambda_{0}>0$ except on the boundary $\Sigma=0$, when $\lambda_{0}=0$, and the other eigenvalues are positive. If $\Gamma=0$ moves into the region $B$ in which $U$ has an odd number of eigenvalues, $\lambda_{0}$ stays negative and no other eigenvalue changes sign. (A similar argument with $\Gamma_{\lambda_{1}}=0$ will prove that $\lambda_{1}$ stays positive as $\Gamma=0$ moves into the region $B$.)

At one point in $D$ there are at least two negative eigenvalues $\lambda_{0}$ and $\lambda_{1}$. Consid- 
eration of the curves $\Gamma_{\lambda_{0}}=0$ and $\Gamma_{\lambda_{1}}=0$, as $(p, q)$ crosses the boundary between $B$ and $D$, shows that on the boundary $\lambda_{0}<0$ and $\lambda_{1}=0$, and in $D$ there are exactly two negative roots.

Thus we may summarize as follows:

(i) In the region $A$ all eigenvalues are positive.

(ii) On the boundary between $A$ and $B$ one eigenvalue is zero and the rest are positive.

(iii) In the region $B$ one eigenvalue is negative and the rest are positive.

(iv) On the boundary between $B$ and $D$ one eigenvalue is negative, one eigenvalue is zero, and the rest are positive.

(v) In $D$ there are two negative eigenvalues and the rest are positive.

The difference scheme (6) will be stable therefore if and only if the values of $p$ and $q$ come from the region $A$ or the boundary between the regions $A$ and $B$.

5. Numerical Results. In order to demonstrate the results of the preceding sections, we considered the problem

$$
\frac{\partial u}{\partial t}=\frac{\partial}{\partial x}\left(\frac{1}{x^{L}} \frac{\partial u}{\partial x}\right)
$$

in the region

$$
[2 \leqq x \leqq 3] \times[t \geqq 0]
$$

subject to the initial conditions

$$
u(x, 0)=x^{L+1}-4, \quad 2 \leqq x \leqq 3,
$$

and the boundary conditions

$$
\begin{aligned}
\partial u / \partial x-p u & =(L+1) 2^{L}-p\left(2^{L+1}-4\right), & & x=2, \\
\partial u / \partial x+q u & =(L+1) 3^{L}+q\left(3^{L+1}-4\right), & & x=3,
\end{aligned}
$$

\begin{tabular}{|c|c|c|c|c|c|}
\hline Problem & (1) & (2) & (3) & (4) & (5) \\
\hline$L=1$ & & & & & \\
\hline$p=$ & 2.00 & -0.20 & -0.36 & -2.00 & -3.00 \\
\hline $\begin{aligned} q & = \\
L & =3\end{aligned}$ & 1.00 & 1.00 & 1.00 & 1.00 & -3.00 \\
\hline$p=$ & 2.00 & -0.10 & -0.18 & -2.00 & -3.00 \\
\hline$q=$ & 1.00 & 1.00 & 1.00 & 1.00 & -3.00 \\
\hline
\end{tabular}

where $L$ was taken equal to 1 and 3 .

The method of Tikhonov and Samarskii was used to solve this problem with $N=20$ and $r=1$ and with the following values of $p$ and $q$ for $L=1$ and $L=3$ :

Table 1 shows the maximum error for values of $K=200(200) 2000$ where $K$ is the number of steps in time. Problems (1), (2), (3) are taken with $(p, q)$ in the region $A$ and are stable; problems (4) and (5) have $(p, q)$ in the regions $B$ and $D$ respectively and are unstable. 


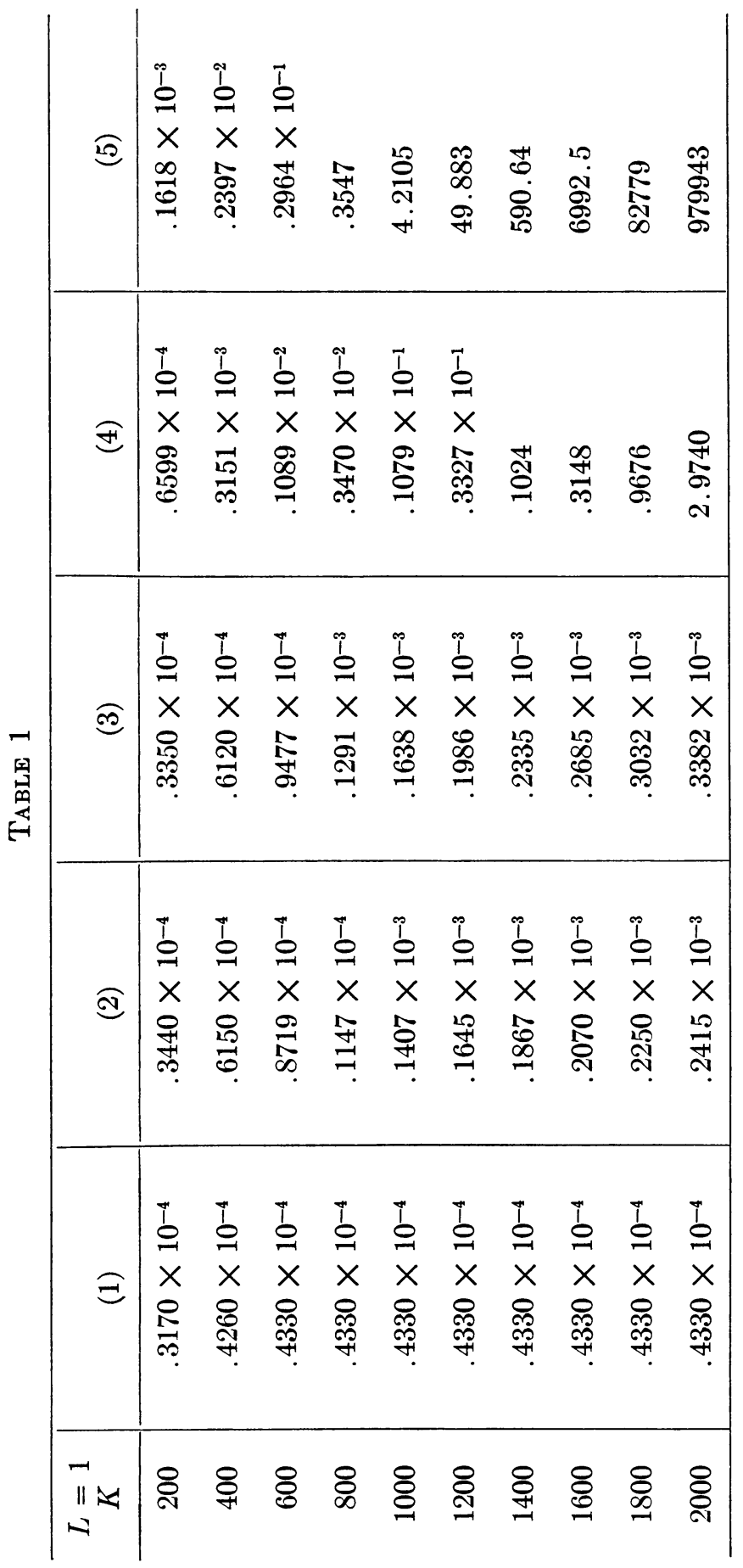




\begin{tabular}{|c|c|c|c|c|c|c|c|c|c|c|}
\hline$\sqrt{20}$ & $\stackrel{\stackrel{ }{\ddagger}}{Ð}$ & 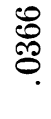 & $\begin{array}{l}\infty \\
\stackrel{2}{0} \\
\end{array}$ & 先 & 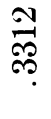 & 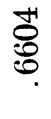 & 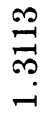 & 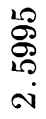 & $\frac{\mathscr{g}}{\dot{g}}$ & 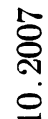 \\
\hline 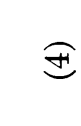 & $\begin{array}{l}8 \\
\varrho\end{array}$ & $\stackrel{10}{\stackrel{0}{0}}$ & $\frac{0}{9}$ & $\stackrel{\infty}{\infty}$ & $\stackrel{20}{\frac{10}{0}}$ & 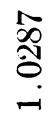 & 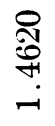 & $\begin{array}{l}\frac{m}{0} \\
\stackrel{0}{0} \\
\text { i }\end{array}$ & $\begin{array}{l}\vec{D} \\
\infty \\
\infty \\
\sim\end{array}$ & $\underset{+}{\stackrel{9}{0}}$ \\
\hline (્) & $\begin{array}{l}8 \\
8 \\
8\end{array}$ & $\begin{array}{l}1 \\
\infty \\
8\end{array}$ & $\stackrel{5}{0}$ & $\stackrel{\stackrel{10}{0}}{0}$ & $\frac{\text { 웅 }}{0}$ & 范 & $\stackrel{\sqrt{0}}{0}$ & $\frac{\Omega}{0}$ & $\stackrel{8}{0}$ & ్ㅇㅇ. \\
\hline$\overparen{(}$ & $\begin{array}{l}\infty \\
\stackrel{8}{8} \\
8\end{array}$ & $\begin{array}{l}\text { मे } \\
\text { ᄋ }\end{array}$ & $\begin{array}{l}\stackrel{0}{0} \\
0\end{array}$ & $\frac{\sigma}{\exists}$ & & $\frac{\sqrt[10]{\sharp}}{0}$ & 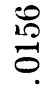 & $\begin{array}{l}0 \\
0 \\
0\end{array}$ & $\stackrel{10}{\stackrel{2}{0}}$ & $\stackrel{10}{\infty}$ \\
\hline 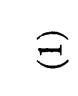 & 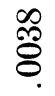 & \& & 오. & 每 & 용 & 今̊ & 今̊ & ڤ్ & 仓ी & $\begin{array}{l}\stackrel{1}{0} \\
\stackrel{8}{8}\end{array}$ \\
\hline $\begin{array}{l}\infty \\
\| \leqslant \\
N\end{array}$ & ఫి & \&̊ & ర్రి & ஓి & \&ి & 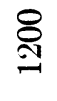 & 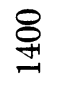 & 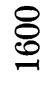 & ర్ & §్రి \\
\hline
\end{tabular}


6. The Differential Equation. Consider Eq. (1) subject to the initial condition (1a) and the boundary conditions

$$
\begin{aligned}
& \partial u / \partial x-p u=0, \quad x=a, \\
& \partial u / \partial x+q u=0, \quad x=b, \quad t \geqq 0 .
\end{aligned}
$$

Let $u(x, t)=X(x) T(t)$ so that

$$
d T / d t=-\lambda T
$$

and

$$
(d / d x)(g(x) d X(x) / d x)=-\lambda X,
$$

where $\lambda$ is a constant and

$$
X^{\prime}(a)-p X(a)=0, \quad X^{\prime}(b)+q X(b)=0 .
$$

Eq. (16) subject to the boundary conditions (17) constitutes a Sturm-Liouville problem for the eigenfunctions $X(x)$ and eigenvalues $\lambda$. The eigenvalues are real, distinct and ordered: $\lambda_{0}<\lambda_{1}<\cdots$ and the corresponding eigenfunctions $X_{i}(x)$ $(i=0,1, \cdots)$ form an orthonormal set in $a \leqq x \leqq b$ [9]. Then the solution of Eq. (1) subject to the initial and boundary conditions is

$$
u(x, t)=\sum_{i=0}^{\infty} A_{i} X_{\imath}(x) e^{-\lambda, t}
$$

where

$$
A_{i}=\int_{a}^{b} X_{i}(x) y(x) d x .
$$

The solution (18) is uniformly bounded as $t \rightarrow \infty$ if and only if all the eigenvalues $\lambda_{i}$ are nonnegative; i.e. if and only if $\lambda_{0} \geqq 0$.

It has been shown [10] that if $p \geqq 0$ and $q \geqq 0$ then $\lambda_{0} \geqq 0$. Thus the solutions of the differential equation are bounded in part of the region $A$ in Fig. 1. We wish to prove that the differential equation has bounded solutions if and only if $(p, q)$ is a point in $A$ or on the boundary between $A$ and $B$; that is if and only if the difference scheme is stable.

The Eq. (16) has a zero eigenvalue if and only if

$$
(d / d x)[g(x)(d X(x) / d x)]=0
$$

i.e. when

$$
X(x)=C \int_{a}^{b} \frac{1}{g(x)} d x+X(a)
$$

where $X(a)$ and $C$ are constants which are obtained from the boundary conditions. The conditions (17) then give the relation

$$
p q \int_{a}^{b} \frac{d x}{g(x)}+\frac{p}{g(b)}+\frac{q}{g(a)}=0
$$


which is equation (13). Thus there is a zero eigenvalue of the Sturm-Liouville problem only on the curve to which $\Sigma$ tends as $N \rightarrow \infty$. By means of an argument similar to the one used on $z(\lambda)$ for the discrete case, it may be shown that $\lambda_{0} \geqq 0$ everywhere in the region $A$ or on the upper branch of the curve (19), where $\lambda_{0}=0$. In addition it may be shown that there is one negative eigenvalue between the branches of the curve (19); one negative and one zero eigenvalue on the lower branch; and two negative eigenvalues in the region inside the lower branch of the curve (19), corresponding to the region $D$.

7. Conclusion. The numerical instability observed in this paper has therefore been traced to the existence of unbounded solutions of the differential equation as $t \rightarrow \infty$. This type of instability will be apparent only if the difference methods are run for large values of $t=n k$. In some problems it is necessary to run the difference methods beyond the stage where initial transients die out (see e.g. [8]) and in such problems asymptotic instability will clearly be of importance. Another important instance of this kind of instability will occur in the iterative solution of systems of equations arising from numerical approximations to Laplace's equation in two or more space variables. It is clear that derivative boundary conditions of the type discussed in this paper will have an effect on the eigenvalues of the matrices occurring in the system and may prevent convergence. This problem, however, will be discussed in a later paper.

The calculations were carried out on the IBM 1620 computer of the University of St. Andrews.

Department of Mathematics

University of St. Andrews

St. Andrews, Fife, Scotland

1. R. S. VARGA, Matrix Iterative Analysis, Prentice-Hall, Englewood Cliffs, N. J., 1962. MR 28 \#1725.

2. M. E. Rose, "On the integration of non-linear parabolic equations by implicit difference methods," Quart. J. Appl. Math., v. 14, 1956, pp. 237-248. MR 19, 65.

3. J. F. GuNN, "The solution of elliptic difference equations by semi-explicit iterative techniques," J. SIAM Ser. B Numer. Anal., v. 2, 1965, pp. 24-45. MR 31 \#4199.

4. A. A. SamarskiI, "Homogeneous difference schemes on non-linear equations of parabolic type," Z. Vycisl. Mat. i. Mat. Fiz., v. 2, 1962, pp. 25-26. (Russian) MR 33 \#5144.

5. I. V. FrYazinov, "On a difference approximation of the boundary conditions for the third boundary-value problem," Z̆. Vyčisl. Mat. $i$. Mat. Fiz., v. 4, 1964, pp. 1106-1112. (Russian) MR $31 \# 895$.

6. P. Keast \& A. R. Mitchell, "On the instability of the Crank Nicholson formula under derivative boundary conditions," Comput. J., v. 9, 1966, pp. 110-114. MR 33 \#5137,

7. A. N. Tikhonov \& A. A. SamarskiI, "Homogeneous difference schemes," Ž. Vyc̀isl. Mat. i. Mat. Fiz., v. 1, 1961, pp. 5-63. (Russian) MR $29 \# 5391$.

8. J. GARY, "A generalization of the Lax-Richtmyer Theorem on finite difference schemes," SIAM J. Numer. Anal., v. 3, 1966, pp. 467-473. MR 34 \#5311.

9. R. Courant \&D. Hilbert, Methods of Mathematical Physics, Vol. 1, Interscience, New York, 1953. MR 16, 426.

10. N. S. Koshlyakov, M. M. Smirnov \& E. B. Gliner, Differential Equations of Mathematical Physics, Amsterdam, North-Holland; Interscience, New York, 1964. MR 31 \#1443. 